This paper is published in Recent Advances in Computational Science and Engineering, eds.

\title{
IMMERSIVE VISUALISATION OF NANO-INDENTATION SIMULATION OF CU
}

\author{
SHUHONG XU, JU LI ${ }^{\dagger}$, CHONGHE LI, FRANK CHAN \\ Institute of High Performance Computing (IHPC), Singapore 117528 \\ E-mail: [xush,lich,chancf]@ihpc.a-star.edu.sg
}

${ }^{\dagger}$ MIT, Massachusetts Avenue, Cambridge, MA02139, USA

E-mail: liju99@mit.edu

\begin{abstract}
This paper introduces the immersive visualisation of nano-indentation simulation of $\mathrm{Cu}$. The molecular dynamics simulation is performed in a system consisting of 326,592 atoms of size $18.6 \times$ $17.0 \times 18.4 \mathrm{~nm}$, under periodic boundary conditions. Based on the coordination number calculation, a practical method for the extraction of "extraordinary" atoms is developed. The simulation has been visualised in the CAVE ${ }^{\mathrm{TM}}$ - an advanced full-immersive virtual environment.
\end{abstract}

\section{Introduction}

With the advent of high performance computing, materials research now embraces another approach: computer simulation. Increasingly materials modelling has taken on the meaning of theory and simulation of materials properties and behaviours. Indeed, in the near future, it is not far-fetched for new materials to be created with enhanced performance, extended service life, acceptable environmental impact and reduced cost. This is realised by exploiting advanced materials modelling and visualisation techniques.

Scientists became aware of the importance of molecular graphics in the mid-1960s [1]. However, Due to the limitations of computer hardware and numerical technology, molecular modelling and visualisation research did not prosper until 1990s. In 1992, Richardson [2] described the kinemage, and supporting programs MAGE and PREKIN. This was the first program that brought molecular visualisation to lots of users. In 1993, Roger Sayle [3] developed a much complete molecular visualisation system RasMol and placed the $\mathrm{C}$ language source code in the public domain. This allowed others to adapt the program to additional types of computers, and to incorporate RasMol's wonderful user interface and renderings into derivative programs. Such derivatives include notably MDL Chime [4] and WebLab [5]. RasMol is widely used throughout the world. However, its as well as PDB file format is not very convenient for large-scale atomistic simulations. So, $\mathrm{Ju} \mathrm{Li}$ [6] developed his own visualisation program atomEye in 1999. More molecular visualisation tools can be found at [7]. One notable phenomenon is that all these systems confine the user to a 2D environment to visualise and interact with 3D molecular structures. This can be very limiting in that the spatial relationships between atoms may be unclear. Virtual reality systems such as $\mathrm{CAVE}^{\mathrm{TM}}$ make $3 \mathrm{D}$ spatial interaction possible. Being fully immersed in a CAVE ${ }^{\mathrm{TM}}$ environment, the user can interact visually, aurally, and tactilely with molecular models in the most natural way. This helps users efficiently extract intrinsic physical properties and gain mechanistic insights from atomistic modelling. Interactive and immersive visualisation of Molecular Dynamics (MD) simulations is still in its infancy, especially for large data. How to efficiently extract useful features within a large-bulk of uninteresting atoms and how to achieve real-time walkthrough speed remains two of the top challenges.

This paper introduces the immersive visualisation of nano-indentation simulation of $\mathrm{Cu}$. A practical method for feature extraction is presented and an integrated approach is 
employed to achieve real-time walkthrough speed. The system has been implemented in a CAVE $^{\mathrm{TM}}$ virtual environment. The paper is structured as follows. Section 2 briefly introduces MD simulation of nano-indentation of $\mathrm{Cu}$. The method for "extraordinary" atom extraction is presented in Section 3. Section 4 introduces the techniques for realtime visualisation. Conclusion and future work is drawn in Section 5.

\section{Nano-Indentation Simulation}

MD simulation of nano-indentation of $\mathrm{Cu}$ is performed in a system consisting of 326,592 atoms of size $18.6 \times 17.0 \times 18.4 \mathrm{~nm}$, under periodic boundary conditions. The (111) surface of the system faces the indentor, which is a blunt cylinder $2.5 \mathrm{~nm}$ in diameter, composed of immobile $\mathrm{Cu}$ atoms, as shown in Fig. 1. The simulation is carried out at $\mathrm{T}=0 \mathrm{~K}$ and $\mathrm{T}$ $=300 \mathrm{~K}$. The EMT potential [8] is used. Fig. 2 shows one configuration [9].

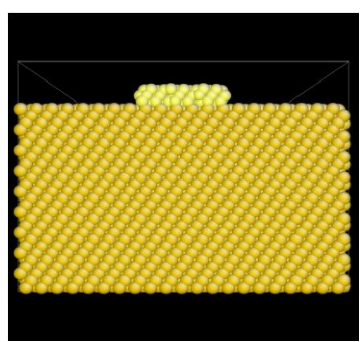

Fig. 1 Nano-indentation simulation setup

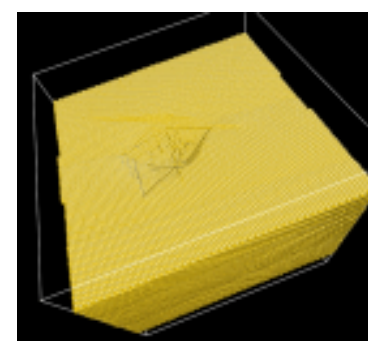

Fig. 2 Nano-indentation of $\mathrm{Cu}$

The MD simulation shows successive events of stress reliefs in variation with indentor depth, thus providing an atomistic link between heterogeneous deformation response and the theoretical strength of the bulk material. For the details, see [9].

\section{Extraction of "Extraordinary" Atoms}

One of the problems of 3D materials simulations is that we may only be interested in a small subset of the data such as dislocations or defects. Often, a feature of interest is embedded within a large-bulk of uninteresting atoms. As a typical example, Fig. 2 shows the surface slip lines caused by nano-indentation. But it cannot reveal internal dislocation information. A bulk view without treatment is not helpful due to the large quantity of atoms.

To extract interesting features, a practical method is to make use of the coordination number. In a perfect crystal lattice, every atom would have the same number of neighbours since every atom is equivalent. However, in a configuration other than a perfect crystal lattice, the above scheme would yield some atoms with different number of nearest neighbours than the others. For perfect crystal $\mathrm{Cu}$ at equilibrium, its crystal structure is FCC and the lattice constant is $\mathrm{a}=0.36078 \mathrm{~nm}$. Each inner atom would initially have twelve nearest neighbours and $4 r=\mathrm{a} \sqrt{2}$, as illustrated in Fig. 3. After indentation, the nearest neighbours of each atom can be found using a cut-off radius $R_{\text {cut }}$. If the distance $d_{\mathrm{i}, \mathrm{j}}$ between arbitrary two atoms $\mathbf{P}_{\mathrm{i}}$ and $\mathbf{P}_{\mathrm{j}}$ is less than $R_{\text {cut }}$, then $\mathbf{P}_{\mathrm{j}}$ is one of $\mathbf{P}_{\mathrm{i}}$ 's nearest neighbours. Obviously, $2 \mathrm{r}<R_{\text {cut }}<$ a, i.e. $0.255110 \mathrm{~nm}<R_{\text {cut }}<0.36078 \mathrm{~nm}$. An enclosing box $\left\{\left[x_{\mathrm{i}}-\Delta \mathrm{x}, x_{\mathrm{i}}+\Delta \mathrm{x}\right],\left[y_{\mathrm{i}}-\Delta \mathrm{y}, y_{\mathrm{i}}+\Delta \mathrm{y}\right],\left[z_{\mathrm{i}}-\Delta \mathrm{z}, z_{\mathrm{i}}+\Delta \mathrm{z}\right]\right\}$ can be used to look for the potential nearest neighbours and speed up the distance calculations, where $\left(x_{i}, y_{i}, z_{i}\right)$ 
are the Cartesian coordinates of $\mathbf{P}_{\mathrm{i}}, \Delta \mathrm{x}, \Delta \mathrm{y}$, and $\Delta \mathrm{z}$ are pre-defined non-negative values. After "ordinary" atom deletion, the "extraordinary" atoms are shown in Fig. 4.

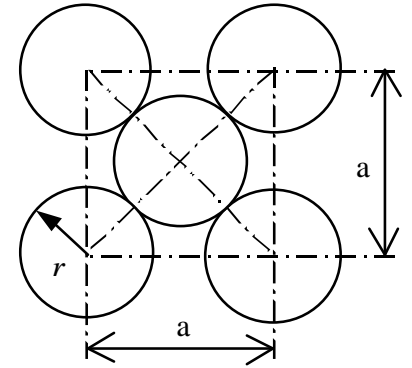

Fig. 3 Crystal structure of perfect $\mathrm{Cu}((001)$ plane)

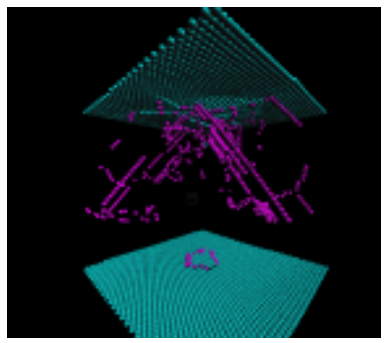

Fig. 4 "Extraordinary" atom extraction

\section{Immersive Visualisation}

Interactive visualisation in an immersive environment has crucial requirement on frame rate (normally should $\geq 15 \mathrm{fps}$ ). To achieve real-time walkthrough, A. Nakano and R. K. Kalia [10] used a multiresolution MD algorithm and the associated data structures to visualise large quantity atoms. In another paper, A. Nakano [11] employed an octree data structure for visibility culling and levels-of-detail control for fast rendering. Theoretically, these techniques will decrease the number of polygons to be displayed. However, accounting for the user perspective requires additional calculations. In my opinion, solving large-scale MD visualisation problem requires an integrated systems approach. On a high level, the following four issues are worth mentioning:

1. Data pre-processing and feature extraction. Generally, a direct rendering of all atoms is not helpful. We are interested in extracting the physically based features that we specify. We can represent these features, if successfully extracted, in an economical way for interactive visualisation.

2. Scalable parallel visualisation. To visualise large-scale MD simulations at the highest possible resolution, we need the processing power and memory of a parallel computer. PC clusters, which have become increasingly popular and affordable, make parallel visualisation an even more attractive approach [12].

3. Optimisation of basic graphics functionality. For molecular visualisation, only very few types of objects in massive quantities, such as spheres (as atoms), cylinders (as bonds), and points (as charge density), need to be rendered. Therefore, very efficient graphics routines might be written and optimised.

4. Visualisation management. There exist some useful techniques for large-scale visualisation, such as visibility culling using octree data structure [13], Level of Detail (LOD) and database paging [14], etc.

We adopt an integrated approach. Before visualisation, MD simulation results are preprocessed and the coordination number of each atom is calculated. According to the purpose of MD simulations, atoms might be divided into three groups according to their significance, i.e. important, normal and less important. For important atoms, we use high quality spheres (more polygons) to represent them. We also extend the LOD technique for navigation. During fast navigation, atoms are rendered in a lower resolution. The system has been implemented in the $\mathrm{CAVE}^{\mathrm{TM}}$ located at IHPC. The host machine is a SGI 
Onyx2 with four InfiniteReality2 graphics pipelines, sixteen R10000 CPUs and eight Gbytes of system RAM. Fig. 5 shows one example.

\section{Conclusion}

The techniques for immersive visualisation of large-scale MD simulations are introduced. Based on the brief introduction to MD simulation of nano-indentation of $\mathrm{Cu}, \mathrm{a}$ practical feature extraction method is presented. This may help materials researchers identify the atoms of interesting and gain mechanistic insights from atomistic modelling, at the same time, reduce rendering burden. To achieve realtime walkthrough in a full-immersive virtual environment, we adopt an integrated approach. Visualisation of MD simulations in the

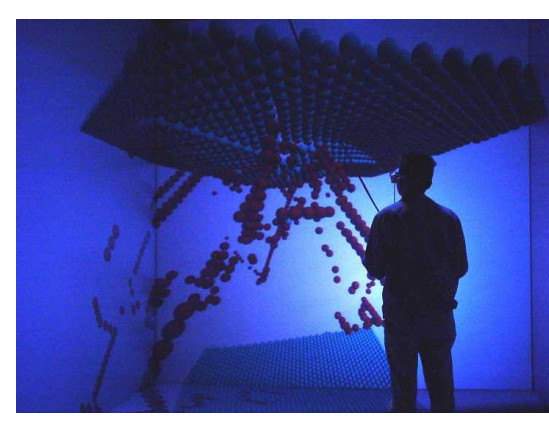

Fig.5 Immersive visualisation in CAVE $\mathrm{CAVE}^{\mathrm{TM}}$ environment provides users a very intuitive way to understand, explore and interact with the microworld. The feedback from our users is very positive. Future work will focus on exploring collaborative visualisation of large-scale MD simulations in tele-immersive environments over broadband networks.

\section{References}

1. Levinthal, Cyrus, "Molecular Model-Building by Computer", Scientific American 214(6):42-52 (1966).

2. D. C. Richardson, J. S. Richardson, "The kinemage: a tool for scientific communication", Protein Science 1, 3-9 (1992).

3. http://www.umass.edu/microbio/rasmol

4. http://www.umass.edu/microbio/chime/

5. http://www.accelrys.com/about/msi.html

6. http://long-march.mit.edu/liju99/Graphics/A/

7. http://molvis.sdsc.edu/visres/index.html

8. K.W. Jacobsen, P. Stoltze, J.K. Norskov, "A semi-empirical effective medium theory for metals and alloys", Surface Science 366, 394 (1996).

9. Ju Li. "Modeling microstructural effects on Deformation Resistance and Thermal Conductivity ”, Ph.D. thesis, MIT, Dept. of Nuclear Engineering (2000).

10. A. Nakano, R. K. kalia, and P. Vashishta, "Scalable Molecular-Dynamics, Visualisation, and Data-Management Algorithms for Materials Simulations", Computing in Science \& Engineering 1(5), 39-47 (1999)

11. A. Nakano, M. E. Bachlechner, etc., "Multiscale Simulation of Nanosystems", Computing in Science \& Engineering 3(4), 56-66 (2001)

12. B. Wylie, C. Pavlakos, etc., "Scalable Rendering on PC Clusters", IEEE Computer Graphics and Applications 21(4), 62-70 (2001)

13. http://www.flipcode.com/tutorials/tut octrees.htm

14. J. Hartman and P. Creek, IRIS Performer Programming Guide, (Silicon Graphics, Inc. 1998) 